\title{
Neutrophils alter epithelial response to Porphyromonas gingivalis in a gingival crevice model
}

\author{
Jessica L. Bondy-Carey ${ }^{1}$, Johnah Galicia ${ }^{2}$, Juhi Bagaitkar ${ }^{3}$, Jan S. Potempa ${ }^{1,4}$, Barbara \\ Potempa $^{1}$, Denis F. Kinane ${ }^{5}$, Florian Veillard ${ }^{1}$, and David A. Scott ${ }^{1,6}$
}

${ }^{1}$ Centre for Oral Health and Systemic Disease, University of Louisville, 501 S. Preston St., Louisville, KY, USA ${ }^{2}$ School of Dentistry, University of North Carolina at Chapel Hill, Chapel Hill, NC, USA ${ }^{3}$ Department of Pediatric Hematology, Washington University, St. Louis, MO, USA ${ }^{4}$ Department of Microbiology, Faculty of Biochemistry, Biophysics and Biotechnology, Jagiellonian University, Krakow, Poland ${ }^{5}$ School of Dental Medicine, University of Pennsylvania, Philadelphia, PA, USA

\section{Summary}

A gingival crevice model (epithelial cell- Porphyromonas gingivalis - neutrophil) was established and used to profile gingipain, matrix metalloproteinase, MMP mediators (NGAL and TIMP-1) and cytokine networks. Smoking is the primary environmental risk factor for periodontitis. Therefore, the influence of cigarette smoke extract (CSE) was also monitored in the same model. $P$. gingivalis alone induced low levels of IL- $1 \beta$ and IL-8 from epithelial cells, but high levels of both cytokines were produced on the addition of neutrophils. CSE-exposure $(100$ and $1000 \mathrm{ng} / \mathrm{ml}$ nicotine equivalency) significantly compromised $P$. gingivalis-induced cytokine secretion (both $\mathrm{p}$ $<0.05)$. P. gingivalis induced impressive secretion of NGAL ( $<0.05)$ which was not influenced by CSE. The influence of CSE on gingipains production was strain-specific. Purified gingipains effectively and rapidly degraded both TIMP-1 and MMP-9. Induction of large amounts of NGAL, degradation of TIMP-1, and increased gingipain activity would each be expected to prolong collagen degradation and promote disease progression. However, gingipains also degrade MMP-9. Thus, $P$. gingivalis exerts a complex influence on the proteolytic balance of a gingival crevice model. CSE-exposure reduces the pro-inflammatory cytokine burden, which may be expected to promote $P$. gingivalis survival. In addition to novel findings that provide mechanistic insight into periodontal disease progression, these results are in keeping with the recognized clinical dogma of decreased inflammation / increased disease in smokers. Thus, this straightforward gingival crevice model is established as a suitable vehicle for the elucidation of mechanisms that contribute to susceptibility to periodontitis.

\section{Keywords}

cytokines; epithelial cells; neutrophils; matrix metalloproteinases; periodontitis; P. gingivalis; tobacco smoke

\section{Introduction}

Periodontitis is a common infectious, chronic inflammatory disease of the supportive structures of the teeth (Detert et al., 2010, Tonetti, 2009). Furthermore, multiple serious

\footnotetext{
${ }^{6}$ Corresponding author: David A. Scott, Centre for Oral Health and Systemic Disease, University of Louisville, 501 S. Preston St., Louisville, KY, USA. Tel: +1-502-852-8905. dascot07@louisville.edu.
} 
systemic sequelae are associated with the establishment of destructive periodontal mucosal lesions, including vascular and pulmonary diseases, pre-term birth, rheumatoid arthritis and diabetes complications (Scannapieco et al., 2010). Histopathological studies and clinical assays have established that, while some periodontal pathogens can invade epithelial cells or cause low level bacteremia (Saito et al., 2009, Castillo et al., 2011), plaque bacteria are, essentially, confined within the gingival crevice where a high density of functional neutrophils are recruited and form a wall between the dental biofilm and the junctional epithelium (Galicia et al., 2009, Scott and Krauss, 2011). Despite this, most prior studies of $P$. gingivalis-host interactions have included epithelial cells or leukocytes, but not both, our own work included (Sandros et al., 2000, Bagaitkar et al., 2009, Rehani et al., 2008).

In order to increase relevance, a multilayer epithelial cell model has been established with which to observe P. gingivalis - host interactions (Dickinson et al., 2011). We have recently described a gingival crevice model in which primary human neutrophils can protect human gingival epithelial cells (HGECs) from $P$. gingivalis-induced apoptosis with the neutrophils themselves exhibiting prolonged longevity (Galicia et al., 2009). The protection afforded to epithelial cells was hypothesized to be partly due to efficient phagocytosis and bacterial killing by neutrophils. Furthermore, bacteria-induced cytokine production, particularly IL1 $\beta$ and IL-8, was increased in the tripartite model relative to the combined cytokine output of epithelial cells and neutrophils stimulated with $P$. gingivalis separately, suggesting a synergistic innate response (Galicia et al., 2009).

Tobacco use may be responsible for the majority of cases of adult periodontitis in the United States (Tomar and Asma, 2000). Smoking leads to a suppression of key inflammatory cytokines in gingival tissues and fluids (Palmer et al., 2005) and the neutrophilic oxidative burst (Palmer et al., 2005, Xu et al., 2008), which are thought to contribute to the established phenomena of reduced overt inflammation and increased susceptibility to infection with $P$. gingivalis and other periodontal pathogens in smokers relative to non-smokers (Palmer et al., 2005, Rehani et al., 2008). Concomitantly, a tobacco-induced endogenous proteaseantiprotease imbalance (Palmer et al., 2005, Gursoy et al., 2010) aligned with virulence mechanisms of established plaque bacteria, such as the gingipains of $P$. gingivalis (Guo et al., 2010), are likely to contribute to the progression of periodontitis in smokers. Of the endogenous proteases, MMPs - the therapeutic target of sub-antimicrobial doses of tetracyclines - are considered of prime importance (Gu et al., 2011).

Therefore, we assessed, in a tripartite gingival crevice model, (a) the production of representative pro-inflammatory cytokines (IL-1 $\beta$ and IL-8); MMPs (MMP-8 and -9); and MMP-modulators (TIMP-1 and NGAL), (b) the effect of cigarette smoke extract (CSE) on these key mediators of inflammation and thus, (c) set out to establish the relevance of a crevicular model with respect to established in vivo phenomena, in order to permit future mechanistic studies. The influence of CSE on P. gingivalis-derived gingipains was also assessed.

\section{Materials and Methods}

\section{Materials}

P. gingivalis strains W83, HG66 and ATCC 33277 were purchased from the American Type Culture Collection (Manassas, VA). Unless otherwise stated, ATCC 33277 was used in all experiments. Gifu Anaerobe Medium [GAM] came from Nissui Pharmaceutical (Tokyo). 2aminoethanol, amphotericin B (fungizone), insulin, 2-mercaptoethanol, penicillin/ streptomycin, Sephadex G-150, sodium selinite, transferrin and Na-p-tosyl-L-lysine chlorometyl ketone (TLCK) were purchased from Sigma-Aldrich Co. (St. Louis, MO). Collagen-coated plates were purchased from BD Biosciences (San Jose, CA). Keratinocyte 
serum-free medium (KSFM), supplements including bovine pituitary extract, 4-12\% NuPage Novex Bis-Tris minigels and Sypro® ruby protein stain came from Invitrogen (Carlsbad, CA). Precision Plus prestained protein standards were bought from Bio-Rad Life Science (Hercules, CA). Acetone, culture plates, dextran and lymphocyte separating medium came from Fisher Scientific (Suwanee, GA). Standard 3R4F reference cigarettes were obtained from Kentucky Tobacco Research and Development Center (Lexington, KY). IL-1 $\beta$ and IL-8 ELISA kits were purchased from eBioscience (San Diego, CA) and Cell Sciences (Canton, MA), respectively, while MMP-8, MMP-9, NGAL and TIMP-1 ELISAs, as well as rMMP-9 and rTIMP-1, came from R\&D Systems (Minneapolis, MN). Gingipain substrates (Rgp: N-a-benzoyl-DL-arginine-p-nitroanilide [BAPNA]; Kgp: acetyl-lysine-p-nitroanilide [ALNA]) were purchased from Sigma and Bachem America, Inc. (Torrance, CA), respectively.

\section{Bacterial culture}

P. gingivalis was grown in Gifu Anaerobic Medium (GAM) anaerobically $\left(80 \% \mathrm{~N}_{2}, 10 \%\right.$ $\mathrm{H}_{2}, 10 \% \mathrm{CO}_{2}$ ) at $37^{\circ} \mathrm{C}$ in a Coy Laboratories anaerobic chamber (Grass Lake, MI), harvested at mid-log phase by centrifugation, washed three times in PBS (pH 7.4), and used immediately.

\section{Gingival epithelial cell isolation and culture}

Primary human gingival epithelial cells (HGECs) were isolated from trypsinated, periodontally healthy gingival tissue biopsies obtained from patients undergoing crownlengthening procedures, as previously described (Guggenheim et al., 2009). Informed and written consent of tissue donors, as approved by the University of Louisville, Institutional Review Board, Human Subjects Protection Program (Study \# 619-03) was obtained. HGEC were seeded in $60-\mathrm{mm}$ plastic tissue culture plates coated with type-I collagen and incubated in $5 \% \mathrm{CO}_{2}$ at $37^{\circ} \mathrm{C}$ using $\mathrm{KSFM}$ medium containing $10 \mu \mathrm{g} \mathrm{ml}^{-1}$ of insulin, $5 \mu \mathrm{g} \mathrm{ml}^{-1}$ of transferrin, $10 \mu \mathrm{M}$ of 2-mercaptoethanol, $10 \mu \mathrm{M}$ of 2-aminoethanol, $10 \mathrm{mM}$ of sodium selenite, $50 \mu \mathrm{g} \mathrm{ml}^{-1}$ of bovine pituitary extract, 100 units ml ${ }^{-1}$ of penicillin/streptomycin and $50 \mathrm{ng} \mathrm{ml}^{-1}$ of fungizone (complete medium). Antibiotic-free medium was used in all experiments involving the use of bacteria.

\section{Conditioning of growth media}

CSE-conditioned growth media (GAM-CSE for prokaryotes and KSFM-CSE for eukaryotes) were prepared by pulling cigarette smoke from standard 3R4F reference cigarettes through medium in $35 \mathrm{ml}$ "drags" every 20 seconds, as previously described (Bagaitkar et al., 2009). Media was sterile filtered $(0.22 \mu \mathrm{m})$; diluted to the required concentration in nicotine equivalents $(0,100$ and $1000 \mathrm{ng} / \mathrm{ml})$, determined by gas-liquid chromatography, as previously described (Fraser et al., 2001); and pH adjusted to $\mathrm{pH}$ 7.2. Nicotine equivalency at physiological concentrations (Fraser et al., 2001) is assumed to similarly dilute the multiple other soluble components of cigarette smoke to relevant levels.

\section{Isolation of primary human neutrophils}

Blood was collected from healthy donors by venipuncture, as approved by the University of Louisville, Institutional Review Board, Human Subjects Protection Program (Study \# 619-03 and \#503.05), and anti-coagulated with acid citrate dextrose. Neutrophils were isolated using lymphocyte separating medium and dextran sedimentation. Neutrophil preparations were routinely $>98 \%$ pure and $>95 \%$ viable. 


\section{Epithelial - neutrophil - P. gingivalis interactions}

HGEC - neutrophil - P. gingivalis interactions were progressed as previously described (Galicia et al., 2009). HGEC monolayers $\left(1 \times 10^{6}\right.$ cells; fourth passage) were overlaid with viable neutrophils $\left(1 \times 10^{6}\right.$ cells $)$ and placed back in the incubator for 30 mins. Live $P$. gingivalis $\left(1 \times 10^{8}\right.$ cells) in KSFM or KSFM supplemented with CSE (100 and $1000 \mathrm{ng} / \mathrm{ml}$ nicotine equivalents) were added to the HGEC-neutrophil combination for 24 hours.

\section{Cytokine profiling}

Cytokine (IL-1 $\beta$ and IL-8) concentrations in $24 \mathrm{hr}$ cell-free supernatants were measured by ELISA, according to the manufacturer's instructions. Plates were read at the appropriate absorbance using a Victor ${ }^{3} 1420$ microplate reader (PerkinElmer, Waltham, MA).

\section{Profiling of matrix metalloproteinases and regulators}

MMP-8, -9, NGAL and TIMP-1 concentrations in $24 \mathrm{hr}$ cell-free supernatants were measured by ELISA, according to the manufacturer's instructions. Plates were read at the appropriate absorbance using a Victor $^{3} 1420$ microplate reader.

\section{Gingipain profiling}

P. gingivalis cultures grown in either GAM or GAM-CSE were normalized for cell number $\left(10^{9}\right.$ cells $\left./ \mathrm{ml}\right)$ using sterile media. Cell-free supernatants were removed and corresponding cell pellets re-suspended in buffer supporting gingipain activity $(0.2 \mathrm{M}$ Tris- $\mathrm{HCl}, 0.1 \mathrm{M} \mathrm{NaCl}$, $5 \mathrm{mM} \mathrm{CaCl}_{2}, 10 \mathrm{mM} \mathrm{L-cysteine,} \mathrm{pH}$ 7.6). Rgp and Kgp activities in cell-free supernatant or associated with whole bacterial cells were determined by enzymatic hydrolysis of $\mathrm{N}-\mathrm{a}-$ benzoyl-DL-arginine- $p$-nitroanilide (BApNA) or acetyl-lysine- $p$-nitroanilide (AL $p \mathrm{NA}$ ) at $1 \mathrm{mM}$ final concentration with absorbance measured at $405 \mathrm{~nm}$ after $3 \mathrm{hr}$, as we have previously described (Potempa and Nguyen, 2007), using a Victor ${ }^{3} 1420$ microplate reader.

\section{Purification of Rgp and Kgp gingipains}

High molecular mass gingipain R (HRgpA) and gingipain K (Kgp) were purified from cellfree medium of $P$. gingivalis $\mathrm{HG66}$, and RgpB from $P$. gingivalis $\mathrm{W} 83$, by acetone precipitation, size-exclusion chromatography using Sephadex G-150, and affinity chromatography on Lysine-Sepharose as we have previously described (Potempa and Nguyen, 2007, Skottrup et al., 2011).

\section{Gingipain activity against TIMP-1 and MMP-9}

TIMP-1 or MMP-9 ( $400 \mu \mathrm{g} / \mathrm{ml}$, final concentration) was incubated with Kgp, RgpB or HRgpA (10 or $100 \mathrm{nM}$ active protease) in $0.1 \mathrm{M}$ Tris buffer $\mathrm{pH} 7.6,2.5 \mathrm{mM} \mathrm{CaCl}_{2}, 75 \mathrm{mM}$ $\mathrm{NaCl}, 0.01 \% \mathrm{NaN} 3$ supplemented with $10 \mathrm{mM} \mathrm{L}$-cysteine at $37^{\circ} \mathrm{C}$ for 3 hours. The reactions were terminated by the addition of TLCK to $4 \mathrm{mM}$ final concentration. The kinetics of TIMP-1 and MMP-9 degradation by gingipains were measured over 180 min, with reactions stopped at the appropriate time points with TLCK. Time-course analyses of TIMP-1 and MMP-9 hydrolysis were performed as above (10 nM active gingipain for MMP-9; $20 \mathrm{nM}$ active gingipain for TIMP-1). Samples were boiled in reducing SDS-PAGE sample buffer, resolved by gradient SDS-PAGE (4-12\% NuPAGE Novex Bis-Tris minigels) and visualized with Sypro Ruby protein stain and a FluorChem HD2 image system (Alpha Innotech, Santa Clara, CA). Molecular masses were determined by comparison to the migration of Precision Plus pre-stained protein standards. 


\section{Statistical analyses}

All data is presented as the mean \pm standard deviation of triplicate experiments, unless otherwise stated. Differences between cytokine, MMP and MMP modulators produced in the gingival crevice model in the presence or absence of CSE were assessed by ANOVA. Differences in gingipains production in the presence and absence of CSE were assessed by ttest. Analyses were performed, and data presented, using Prism v5.02 and InStat v3.06 (Graphpad Software Inc., La Jolla, CA).

\section{Results}

\section{Induction of cytokines by $\boldsymbol{P}$. gingivalis in a gingival crevice model}

Human gingival epithelial cells were stimulated with $P$. gingivalis in the presence or absence of primary human neutrophils and CSE. As is shown in Figure 1, cytokine production varied dramatically depending on the constituents of the crevicular crevice model. The presence of all three components (HGEC-Neutrophil- $P$. gingivalis) was required for meaningful detection of IL-1 $\beta$ and IL-8, while CSE exposure significantly dampened pro-inflammatory cytokine production.

\section{Induction of MMPs and MMP modulators by P. gingivalis in a gingival crevice model}

As presented in Figure 2, while both neutrophils and epithelial cells are sources of large concentrations of MMP-9, neutrophils are the sole source of MMP-8 in the gingival crevice model. Levels of epithelial cell-derived MMP-9 are reduced in the presence of $P$. gingivalis, presumed to be due to proteolytic degradation, but significantly increased when HGEC, neutrophils and $P$. gingivalis are all present.

Neutrophils produced striking levels of the MMP-stabilizing molecule, NGAL, as shown in Figure 3. Epithelial cells produced significant amounts of the MMP-inhibitor, TIMP-1. Levels of TIMP-1 are reduced in the presence of $P$. gingivalis, again, presumed to be, due to proteolytic degradation. Neutrophils do not produce TIMP-1 (Opdenakker et al., 2001). Therefore, neutrophils were able to partially protect epithelial-derived TIMP-1 in the crevicular crevice model. Interestingly, CSE further protected secreted epithelial cellderived TIMP-1 in a dose-dependent manner.

\section{CSE induces $P$. gingivalis gingipain production}

As gingipains are key virulence factors of $P$. gingivalis that can promote host tissue destruction as well as degrading multiple mediators of the innate and adaptive immune responses, we next set out to establish if CSE influenced gingipain surface expression or secretion in two of the most commonly employed laboratory strains, ATCC 33277 and W83. CSE exposure suppressed cell-bound Kgp and Rgp gingipain production in $P$. gingivalis ATCC 33277, as shown in Figures 4A and C. On the other hand, CSE augmented cellbound, Kgp and Rgp gingipain production in P. gingivalis W83 (Figure 4B). The effect of CSE exposure on the concentration of cell-free gingipains was not so impressive.

\section{Gingipains degrade TIMP-1 and MMP-9}

In gingival crevicular fluid collected from sites colonized with $P$. gingivalis gingipains are present at concentrations up to $100 \mathrm{nM}$ (Guentsch et al., 2011) and may affect balance of MMP and MMP-modulators in the gingival crevice and periodontal pockets. Therefore, to assess if gingipains can degrade MMP-9 and TIMP-1 we have incubated these proteins with RgpA, HRgpA and Kgp. As shown in Figure 5A TIMP-1 was totally degraded without any discrete cleavage products by Kgp and HRgpA applied at 10nM and 100nM concentrations. $\mathrm{RgpB}$ was somehow less effective in TIMP-1 digestion then other gingipains and after $3 \mathrm{~h}$ 
incubation with $10 \mathrm{nM}$ RgpB some intact inhibitor was still observed in the gel (Figures $5 \mathrm{~A}$ and C). Also recombinant MMP-9 (rMMP-9), which occurred predominantly in the $92 \mathrm{kDa}$ latent form with a smaller amount of an $82 \mathrm{kDa}$ isoform, assumed to be active MMP-9 was totally degraded by any gingipain at $100 \mathrm{nM}$ concentration. At this gingipain concentration only the protease-derived bands were observed in gels after $3 \mathrm{~h}$ incubation (Figures $5 \mathrm{~A}$ and B). Conversely to TIMP-1 digestion, incubation of rMMP-9 with gingipains at $10 \mathrm{nM}$ concentration released a discrete metalloprotease-derived cleavage products clearly visible after $3 \mathrm{~h}$. At this concentration of gingipains cleaved rMMP-9 in time-dependent manner releasing several discrete products (Figure 5D). Together this result argues that in vivo balance of MMP and MMP-modulators can be efficiently altered by bacteria-derived proteases.

\section{Discussion}

We have recently published some of the first data describing combinatorial interactions between the histopathologically dominant components of the diseased periodontium bacteria ( $P$. gingivalis), neutrophils, and epithelial cells (Galicia et al., 2009). Here we show that, while HGECs respond to oral bacteria in a manner that is considered critical in initiating the immune response, the introduction of neutrophils dramatically alters the dynamics of inflammation, as reflected in a surge in the production of cytokine and endogenous proteolysis-related factors. This likely occurs primarily due to both the release of inflammatory mediators from neutrophils but, perhaps, also due to the stabilizing effect of neutrophils on gingipain-exposed HGECs (Galicia et al., 2009).

MMP-8 and MMP-9 have both been reported to reflect the inflammatory burden in periodontal tissues and, thus, to represent biomarkers of periodontal disease (Leppilahti et al., 2011, Ramseier et al., 2009). Gingival crevicular modeling has permitted a number of novel insights into perturbations of the protease-antiprotease balance. Detection of total MMP-8 in supernatants of unstimulated or $P$. gingivalis-exposed HGECs was minimal. On the introduction of neutrophils, however, MMP-8 was abundant. MMP-8 production was increased and decreased at low and high CSE doses, respectively, whereas MMP-9 production was not influenced by exposure to CSE. Interestingly, $P$. gingivalis reduced the burden of HGEC-derived MMP-9. Gingipains, which represent the bulk of the extensive proteolytic activity ascribed to $P$. gingivalis, are known to degrade multiple host proteins, including transferrin, defensins, complement proteins and cytokines (Guo et al., 2010, Mezyk-Kopec et al., 2005). Recently, P. gingivalis has been suggested to activate MMP-9 by undefined mechanisms (Zhou et al., 2011), while others have shown that dental plaque taken from periodontitis patients can convert MMP-9 into lower molecular mass forms (Ding et al., 1995). We show, for the first time, that Kgp, RgpA and RgpB are each capable of the rapid and effective degradation of MMP-9. It should be noted that the considerable total MMP-9 released by neutrophils in the gingival crevicular model increases the overall MMP-9 burden, suggesting that the MMP-9-degrading capacity of the gingipains is saturated or blocked.

NGAL is a neutrophil granule protein that covalently interacts with and, subsequently, stabilizes MMP-9 (Yan et al., 2001). We report the novel finding that NGAL was released by neutrophils at impressive levels in the crevicular model. As there are some reports of epithelial-derived NGAL, the source of NGAL in mucosal inflammatory diseases has been debated (Eagan et al., 2010). In our tripartite model, neutrophils are clearly the major contributors to $P$. gingivalis-induced NGAL and the possibility of significant gingipainmediated NGAL degradation was not suggested by the data. This neutrophil-derived NGAL would be expected to prolong MMP-9 activity, augmenting to the collagenolytic activity that is a hallmark of periodontitis. As well as prolonging MMP-9 activity, NGAL takes part in 
the anti-bacterial iron depletion strategy of the innate immune system through its ability to tightly bind to siderophores of the catecholate type (Goetz et al., 2002) and NGAL may also protect the host against reactive oxygen species (Roudkenar et al., 2008).

TIMP-1, the major endogenous MMP inhibitor, is thought not to be produced by neutrophils (Opdenakker et al., 2001). HGECs, therefore, unsurprisingly represented the sole source of TIMP-1 in the current system. We have shown for the first time that, similarly to MMP-9, $P$. gingivalis abrogated HGEC-derived TIMP-1, and the addition of neutrophils was able to only partially restore TIMP-1 concentrations. Therefore, we went on to establish that Kgp, RgpA and RgpB are each capable of the rapid and effective degradation of TIMP-1. Again, TIMP-1 degradation would be expected to augment MMP-mediated collagenolytic activity and abet the progression of periodontitis. These data are in keeping with a prior report that whole $P$. gingivalis can degrade TIMP-1 by an unidentified mechanism (Grenier and Mayrand, 2001).

W83 (CSE upregulates gingipain production) is a high virulence strain, relative to 33277 (Lin et al., 2009, Naito et al., 2008). W83 and 33277 both express Kgp, HRgpA and HRgpB. While the C-terminal hemagluttinin /adhesion 3/4 domain junction differs between the Kgp of W83 and 33277, this protein region is non-catalytic and not involved in gingipain processing or transport (Li et al., 2010, Sztukowska et al., 2004) and, thus, is unlikely to explain the differential gingipain response to CSE. Thus, the mechanisms underlying strainspecific variation in cell-associated gingipain activity upon CSE exposure remain unclear.

Concentrations of IL- 8 and IL- $1 \beta$ in gingival crevicular fluid are known to correlate with periodontal disease severity (Ertugrul et al., 2012). Multiple oral bacteria, in both planktonic and biofilm form, are known to induce IL- $1 \beta$ and IL-8, and other pro-inflammatory cytokines, from human oral epithelial cells (Peyyala et al., 2012). Neutrophils are also known to secrete large amounts of IL-8 and IL-1 $\beta$ (Cassatella, 1995). IL-1 and IL-8 levels detected in 24 hour HGEC supernatants upon stimulation with $P$. gingivalis alone were low, but similar to our prior report using live P. gingivalis (Stathopoulou et al., 2009).

Significantly higher cytokine levels were found on HGEC stimulation with dead $P$. gingivalis or with gingipain deficient mutants (Stathopoulou et al., 2009). In the tripartite gingival crevice model, it appears that neutrophils are the primary source IL- 8 and IL- $1 \beta$, although it cannot be ruled out that neutrophils induce IL- 8 and IL- $1 \beta$ from epithelial cells in a manner that $P$. gingivalis alone does not. Alternatively, $P$. gingivalis-derived proteases may be sufficient to degrade both IL- $1 \beta$ and IL-8 produced by $P$. gingivalis-stimulated epithelial cells but not by the combination of neutrophils and epithelial cells.

Several studies have shown that pro-inflammatory cytokines, including IL-1 $\beta$ and IL- 8 , are reduced in the gingival crevicular fluid of smokers with periodontal diseases compared to diseased non-smokers (Tymkiw et al., 2011, Rawlinson et al., 2003, Palmer et al., 2005). Mechanistically, it is known that smoke exposure alters the activity of multiple $P$. gingivalis genes and the expression levels of several membrane proteins that result in a lower inflammatory potential of this key pathogen (Bagaitkar et al., 2011, Bagaitkar et al., 2010, Bagaitkar et al., 2009). Similarly, it is known that the result of nicotine, and the major human nicotine metabolite, cotinine, engagement of innate cell a 7-nicotinic acetylcholine receptors is the activation of the cholinergic anti-inflammatory and augmentation of the endogenous Akt / GSK-3 $\beta$ anti-inflammatory pathways (Rehani et al., 2008, Martin et al., 2005, Tracey, 2007). Thus, cytokine profiles in the tripartite model are in keeping with established clinical and mechanistic phenomena. Furthermore, the reduced inflammatory response to $P$. gingivalis upon CSE exposure is likely to promote $P$. gingivalis survival. Again, this is in keeping with multiple studies have shown that, relative to non-smokers, smokers are more likely to be infected with $P$. gingivalis, to be infected with higher numbers 
of this bacterium, and to exhibit more persistent infection by this pathogen (Haffajee and Socransky, 2001, Zambon et al., 1996, Eggert et al., 2001, Kamma et al., 1999). Importantly, then, exploitation of crevicular modeling will facilitate a deeper mechanistic understanding of the immune suppression that occurs in the periodontia of smokers and may well have relevance to the general increase in susceptibility to bacterial infections in tobacco users (Bagaitkar et al., 2008).

While MMPs are known to contribute to the destruction of periodontal tissues in smokers and non-smokers alike (Leppilahti et al., 2011, Ramseier et al., 2009), the balance between MMPs and endogenous mediators has consistently been shown to be altered in cigarette smokers (Gursoy et al., 2010, Heikkinen et al., 2010, Liede et al., 1999). Recently, Mouzakiti et al have shown that TIMP-1 mRNA levels in human periodontal tissues removed from smoker subjects with untreated chronic periodontitis are higher in smokers than in non-smokers (Mouzakiti et al., 2011). Several studies have reported reduced MMP-8 concentrations in oral fluids of smokers with periodontitis compared to non-smoker controls (Heikkinen et al., 2011, Heikkinen et al., 2010, Liede et al., 1999, Mantyla et al., 2006), although tissue levels of MMP-8 may be increased (Liu et al., 2006). Thus, the data generated by our in vitro model of the gingival crevice reflect the reported in vivo data, strengthening the suitability of our model for the mechanistic dissection of innate-pathogen interactions in periodontal diseases.

The influence of tobacco smoke exposure on gingipains production has, to the best of our knowledge, not been previously addressed. We show that cell-bound Rgp and Kgp are decreased in CSE-treated $P$. gingivalis ATCC 33277, the type strain, relative to control bacteria, whereas both Rgp and Kgp are significantly upregulated in the highly virulent $P$. gingivalis W83 strain. Thus, the influence of tobacco smoke on gingipain production may be strain-specific.

While the potential for variation in results produced by this model due to genetic differences in primary cell isolates must be acknowledged, it will be possible to incorporate primary cells phenotyped for key innate receptors, such as TLR polymorphisms (Kinane et al., 2006).

In summary, the current findings underscore the importance of neutrophils in modifying the responses of primary gingival epithelial cells to bacterial insults. Rgp and Kgp gingipains are capable of degrading MMP-9 and TIMP-1. Furthermore, CSE-exposure exerts multiple influences on the gingival crevice model consistent with the promotion of $P$. gingivalis infection and established mechanistic phenomena and clinical observations. Thus, the potential utility of the gingival crevice model for mechanistic studies at the cellular and molecular levels is established. Exploitation of the model may help identify therapeutic targets for the treatment of tobacco-induced periodontitis and other chronic inflammatory mucosal diseases. Indeed, this initial publication has already identified several novel mechanisms likely to contribute to periodontal disease progression including $P$. gingivalisinduced NGAL secretion, $P$. gingivalis-induced, gingipain-mediated MMP-9 and TIMP-1 degradation, and CSE-induced alterations to gingipain production.

\section{Acknowledgments}

This work was kindly supported by the National Institute of Dental and Craniofacial Research: DE017680 (DAS), DE019826 (DAS), DE017384 (DFK) and DE09671 (JP). 


\section{References}

Bagaitkar J, Daep CA, Patel CK, Renaud DE, Demuth DR, Scott DA. Tobacco smoke augments Porphyromonas gingivalis - Streptococcus gordonii biofilm formation. PLOS One. 2011; 6:e27386. [PubMed: 22110637]

Bagaitkar J, Demuth DR, Daep CA, Renaud DE, Pierce DL, Scott DA. Tobacco upregulates P. gingivalis fimbrial proteins which induce TLR2 hyposensitivity. PLoS One. 2010; 5:e9323. [PubMed: 20454607]

Bagaitkar J, Demuth DR, Scott DA. Increased susceptibility to bacterial infections in tobacco smokers. Tobacco Induced Diseases. 2008; 4

Bagaitkar J, Scott DA, Williams LR, Renaud DE, Daep C, Martin M, Demuth DR. Tobacco-induced alterations to Porphyromonas gingivalis-host interactions. Environmental Microbiology. 2009; 11:1242-1253. [PubMed: 19175666]

Cassatella MA. The production of cytokines by polymorphonuclear neutrophils. Immunol Today. 1995; 16:21-26. [PubMed: 7880385]

Castillo DM, Sanchez-Beltran MC, Castellanos JE, Sanz I, Mayorga-Fayad I, Sanz M, Lafaurie GI. Detection of specific periodontal microorganisms from bacteraemia samples after periodontal therapy using molecular-based diagnostics. J Clin Periodontol. 2011; 38:418-427. [PubMed: 21392048]

Detert J, Pischon N, Burmester GR, Buttgereit F. The association between rheumatoid arthritis and periodontal disease. Arthritis Res Ther. 2010; 12:218. [PubMed: 21062513]

Dickinson BC, Moffatt CE, Hagerty D, Whitmore SE, Brown TA, Graves DT, Lamont RJ. Interaction of oral bacteria with gingival epithelial cell multilayers. Mol Oral Microbiol. 2011; 26:210-220. [PubMed: 21545698]

Ding Y, Uitto VJ, Firth J, Salo T, Haapasalo M, Konttinen YT, Sorsa T. Modulation of host matrix metalloproteinases by bacterial virulence factors relevant in human periodontal diseases. Oral Dis. 1995; 1:279-286. [PubMed: 8705837]

Eagan TM, Damas JK, Ueland T, Voll-Aanerud M, Mollnes TE, Hardie JA, et al. Neutrophil gelatinase-associated lipocalin: a biomarker in COPD. Chest. 2010; 138:888-895. [PubMed: 20495108]

Eggert FM, McLeod MH, Flowerdew G. Effects of smoking and treatment status on periodontal bacteria: evidence that smoking influences control of periodontal bacteria at the mucosal surface of the gingival crevice. Journal of Periodontology. 2001; 72:1210-1220. [PubMed: 11577953]

Ertugrul AS, Sahin H, Dikilitas A, Alpaslan N, Bozoglan A. Comparison of CCL28, interleukin-8, interleukin-1beta and tumor necrosis factor-alpha in subjects with gingivitis, chronic periodontitis and generalized aggressive periodontitis. J Periodontal Res. 2012

Fraser HS, Palmer RM, Wilson RF, Coward PY, Scott DA. Elevated systemic concentrations of soluble ICAM-1 (sICAM) are not reflected in the gingival crevicular fluid of smokers with periodontitis. J Dent Res. 2001; 80:1643-1647. [PubMed: 11597025]

Galicia JC, Benakanakere MR, Stathopoulou PG, Kinane DF. Neutrophils rescue gingival epithelial cells from bacterial-induced apoptosis. J Leukoc Biol. 2009; 86:181-186. [PubMed: 19389800]

Goetz DH, Holmes MA, Borregaard N, Bluhm ME, Raymond KN, Strong RK. The neutrophil lipocalin NGAL is a bacteriostatic agent that interferes with siderophore-mediated iron acquisition. Mol Cell. 2002; 10:1033-1043. [PubMed: 12453412]

Grenier D, Mayrand D. Inactivation of tissue inhibitor of metalloproteinases-1 (TIMP-1) by Porphyromonas gingivalis. FEMS Microbiol Lett. 2001; 203:161-164. [PubMed: 11583842]

Gu Y, Lee HM, Sorsa T, Salminen A, Ryan ME, Slepian MJ, Golub LM. Non-antibacterial tetracyclines modulate mediators of periodontitis and atherosclerotic cardiovascular disease: A mechanistic link between local and systemic inflammation. Pharmacol Res. 2011; 64:573-579. [PubMed: 21771657]

Guentsch A, Kramesberger M, Sroka A, Pfister W, Potempa J, Eick S. Comparison of gingival crevicular fluid sampling methods in patients with severe chronic periodontitis. J Periodontol. 2011; 82:1051-1060. [PubMed: 21235330] 
Guggenheim B, Gmur R, Galicia JC, Stathopoulou PG, Benakanakere MR, Meier A, et al. In vitro modeling of host-parasite interactions: the 'subgingival' biofilm challenge of primary human epithelial cells. BMC Microbiol. 2009; 9:280. [PubMed: 20043840]

Guo Y, Nguyen KA, Potempa J. Dichotomy of gingipains action as virulence factors: from cleaving substrates with the precision of a surgeon's knife to a meat chopper-like brutal degradation of proteins. Periodontol 2000. 2010; 54:15-44. [PubMed: 20712631]

Gursoy UK, Kononen E, Pradhan-Palikhe P, Tervahartiala T, Pussinen PJ, Suominen-Taipale L, Sorsa T. Salivary MMP-8, TIMP-1, and ICTP as markers of advanced periodontitis. J Clin Periodontol. 2010; 37:487-493. [PubMed: 20507371]

Haffajee AD, Socransky SS. Relationship of cigarette smoking to the subgingival microbiota. J Clin Periodontol. 2001; 28:377-388. [PubMed: 11350499]

Heikkinen AM, Pitkaniemi J, Kari K, Pajukanta R, Elonheimo O, Koskenvuo M, Meurman JH. Effect of teenage smoking on the prevalence of periodontal bacteria. Clin Oral Investig. 2011

Heikkinen AM, Sorsa T, Pitkaniemi J, Tervahartiala T, Kari K, Broms U, et al. Smoking affects diagnostic salivary periodontal disease biomarker levels in adolescents. J Periodontol. 2010; 81:1299-1307. [PubMed: 20450405]

Kamma JJ, Nakou M, Baehni PC. Clinical and microbiological characteristics of smokers with early onset periodontitis. J Periodontal Res. 1999; 34:25-33. [PubMed: 10086883]

Kinane DF, Shiba H, Stathopoulou PG, Zhao H, Lappin DF, Singh A, et al. Gingival epithelial cells heterozygous for Toll-like receptor 4 polymorphisms Asp299Gly and Thr399ile are hyporesponsive to Porphyromonas gingivalis. Genes Immun. 2006; 7:190-200. [PubMed: 16437123]

Leppilahti JM, Ahonen MM, Hernandez M, Munjal S, Netuschil L, Uitto VJ, et al. Oral rinse MMP-8 point-of-care immuno test identifies patients with strong periodontal inflammatory burden. Oral Dis. 2011; 17:115-122. [PubMed: 20659259]

Li N, Yun P, Nadkarni MA, Ghadikolaee NB, Nguyen KA, Lee M, et al. Structure determination and analysis of a haemolytic gingipain adhesin domain from Porphyromonas gingivalis. Mol Microbiol. 2010; 76:861-873. [PubMed: 20233299]

Liede KE, Haukka JK, Hietanen JH, Mattila MH, Ronka H, Sorsa T. The association between smoking cessation and periodontal status and salivary proteinase levels. J Periodontol. 1999; 70:1361-1368. [PubMed: 10588500]

Lin L, Li C, Liu J, Zhang D, Zhao J, Kou Y, et al. Virulence genes of Porphyromonas gingivalis W83 in chronic periodontitis. Acta Odontol Scand. 2009; 67:258-264. [PubMed: 22443638]

Liu KZ, Hynes A, Man A, Alsagheer A, Singer DL, Scott DA. Increased local matrix metalloproteinase- 8 expression in the periodontal connective tissues of smokers with periodontal disease. Biochim Biophys Acta. 2006; 1762:775-780. [PubMed: 16928431]

Mantyla P, Stenman M, Kinane D, Salo T, Suomalainen K, Tikanoja S, Sorsa T. Monitoring periodontal disease status in smokers and nonsmokers using a gingival crevicular fluid matrix metalloproteinase-8-specific chair-side test. J Periodontal Res. 2006; 41:503-512. [PubMed: 17076774]

Martin M, Rehani K, Jope RS, Michalek SM. Toll-like receptor-mediated cytokine production is differentially regulated by glycogen synthase kinase 3. Nat Immunol. 2005; 6:777-784. [PubMed: 16007092]

Mezyk-Kopec R, Bzowska M, Potempa J, Jura N, Sroka A, Black RA, Bereta J. Inactivation of membrane tumor necrosis factor alpha by gingipains from Porphyromonas gingivalis. Infect Immun. 2005; 73:1506-1514. [PubMed: 15731048]

Mouzakiti E, Pepelassi E, Fanourakis G, Markopoulou C, Tseleni-Balafouta S, Vrotsos I. The effect of smoking on the mRNA expression of MMPs and TIMP-1 in untreated chronic periodontitis patients: a cross-sectional study. J Periodontal Res. 2011; 46:576-583. [PubMed: 21732946]

Naito M, Hirakawa H, Yamashita A, Ohara N, Shoji M, Yukitake H, et al. Determination of the genome sequence of Porphyromonas gingivalis strain ATCC 33277 and genomic comparison with strain W83 revealed extensive genome rearrangements in P. gingivalis. DNA Res. 2008; 15:215225. [PubMed: 18524787] 
Opdenakker G, Van den Steen PE, Dubois B, Nelissen I, Van Coillie E, Masure S, et al. Gelatinase B functions as regulator and effector in leukocyte biology. J Leukoc Biol. 2001; 69:851-859. [PubMed: 11404367]

Palmer RM, Wilson RF, Hasan AS, Scott DA. Mechanisms of action of environmental factors-tobacco smoking. J Clin Periodontol. 2005; 32(Suppl 6):180-195. [PubMed: 16128837]

Peyyala R, Kirakodu SS, Novak KF, Ebersole JL. Oral microbial biofilm stimulation of epithelial cell responses. Cytokine. 2012; 58:65-72. [PubMed: 22266273]

Potempa J, Nguyen KA. Purification and characterization of gingipains. Curr Protoc Protein Sci. 2007 Chapter 21, Unit 2120.

Ramseier CA, Kinney JS, Herr AE, Braun T, Sugai JV, Shelburne CA, et al. Identification of pathogen and host-response markers correlated with periodontal disease. J Periodontol. 2009; 80:436-446. [PubMed: 19254128]

Rawlinson A, Grummitt JM, Walsh TF, Ian Douglas CW. Interleukin 1 and receptor antagonist levels in gingival crevicular fluid in heavy smokers versus non-smokers. J Clin Periodontol. 2003; 30:42-48. [PubMed: 12702110]

Rehani K, Scott DA, Renaud D, Hamza H, Williams LR, Wang H, Martin M. Cotinine-induced convergence of the cholinergic and PI3 kinase-dependent anti-inflammatory pathways in innate immune cells. Biochim Biophys Acta. 2008; 1783:375-382. [PubMed: 18178163]

Roudkenar MH, Halabian R, Ghasemipour Z, Roushandeh AM, Rouhbakhsh M, Nekogoftar M, et al. Neutrophil gelatinase-associated lipocalin acts as a protective factor against $\mathrm{H}(2) \mathrm{O}(2)$ toxicity. Arch Med Res. 2008; 39:560-566. [PubMed: 18662586]

Saito A, Inagaki S, Ishihara K. Differential ability of periodontopathic bacteria to modulate invasion of human gingival epithelial cells by Porphyromonas gingivalis. Microb Pathog. 2009; 47:329-333. [PubMed: 19818393]

Sandros J, Karlsson C, Lappin DF, Madianos PN, Kinane DF, Papapanou PN. Cytokine responses of oral epithelial cells to Porphyromonas gingivalis infection. J Dent Res. 2000; 79:1808-1814. [PubMed: 11077999]

Scannapieco FA, Dasanayake AP, Chhun N. Does periodontal therapy reduce the risk for systemic diseases? Dent Clin North Am. 2010; 54:163-181. [PubMed: 20103479]

Scott DA, Krauss JL. Neutrophils in periodontal inflammation. Frontiers in Oral Biology. 2011; 15:56-83.

Skottrup PD, Leonard P, Kaczmarek JZ, Veillard F, Enghild JJ, O’Kennedy R, et al. Diagnostic evaluation of a nanobody with picomolar affinity toward the protease RgpB from Porphyromonas gingivalis. Anal Biochem. 2011; 415:158-167. [PubMed: 21569755]

Stathopoulou PG, Benakanakere MR, Galicia JC, Kinane DF. The host cytokine response to Porphyromonas gingivalis is modified by gingipains. Oral Microbiol Immunol. 2009; 24:11-17. [PubMed: 19121064]

Sztukowska M, Sroka A, Bugno M, Banbula A, Takahashi Y, Pike RN, et al. The C-terminal domains of the gingipain $\mathrm{K}$ polyprotein are necessary for assembly of the active enzyme and expression of associated activities. Mol Microbiol. 2004; 54:1393-1408. [PubMed: 15554977]

Tomar SL, Asma S. Smoking-attributable periodontitis in the United States: findings from NHANES III. National Health and Nutrition Examination Survey. J Periodontol. 2000; 71:743-751. [PubMed: 10872955]

Tonetti MS. Periodontitis and risk for atherosclerosis: an update on intervention trials. J Clin Periodontol. 2009; 36(Suppl 10):15-19. [PubMed: 19432627]

Tracey KJ. Physiology and immunology of the cholinergic antiinflammatory pathway. J Clin Invest. 2007; 117:289-296. [PubMed: 17273548]

Tymkiw KD, Thunell DH, Johnson GK, Joly S, Burnell KK, Cavanaugh JE, et al. Influence of smoking on gingival crevicular fluid cytokines in severe chronic periodontitis. J Clin Periodontol. 2011; 38:219-228. [PubMed: 21198766]

Xu M, Scott JE, Liu KZ, Bishop HR, Renaud DE, Palmer RM, et al. The influence of nicotine on granulocytic differentiation - inhibition of the oxidative burst and bacterial killing and increased matrix metalloproteinase-9 release. BMC Cell Biol. 2008; 9:19. [PubMed: 18412948] 
Yan L, Borregaard N, Kjeldsen L, Moses MA. The high molecular weight urinary matrix metalloproteinase (MMP) activity is a complex of gelatinase B/MMP-9 and neutrophil gelatinaseassociated lipocalin (NGAL). Modulation of MMP-9 activity by NGAL. J Biol Chem. 2001; 276:37258-37265. [PubMed: 11486009]

Zambon JJ, Grossi SG, Machtei EE, Ho AW, Dunford R, Genco RJ. Cigarette smoking increases the risk for subgingival infection with periodontal pathogens. J Periodontol. 1996; 67:1050-1054. [PubMed: 8910822]

Zhou J, Zhang J, Chao J. Porphyromonas gingivalis promotes monocyte migration by activating MMP-9. J Periodontal Res. 2012; 47:236-242. [PubMed: 22035412] 

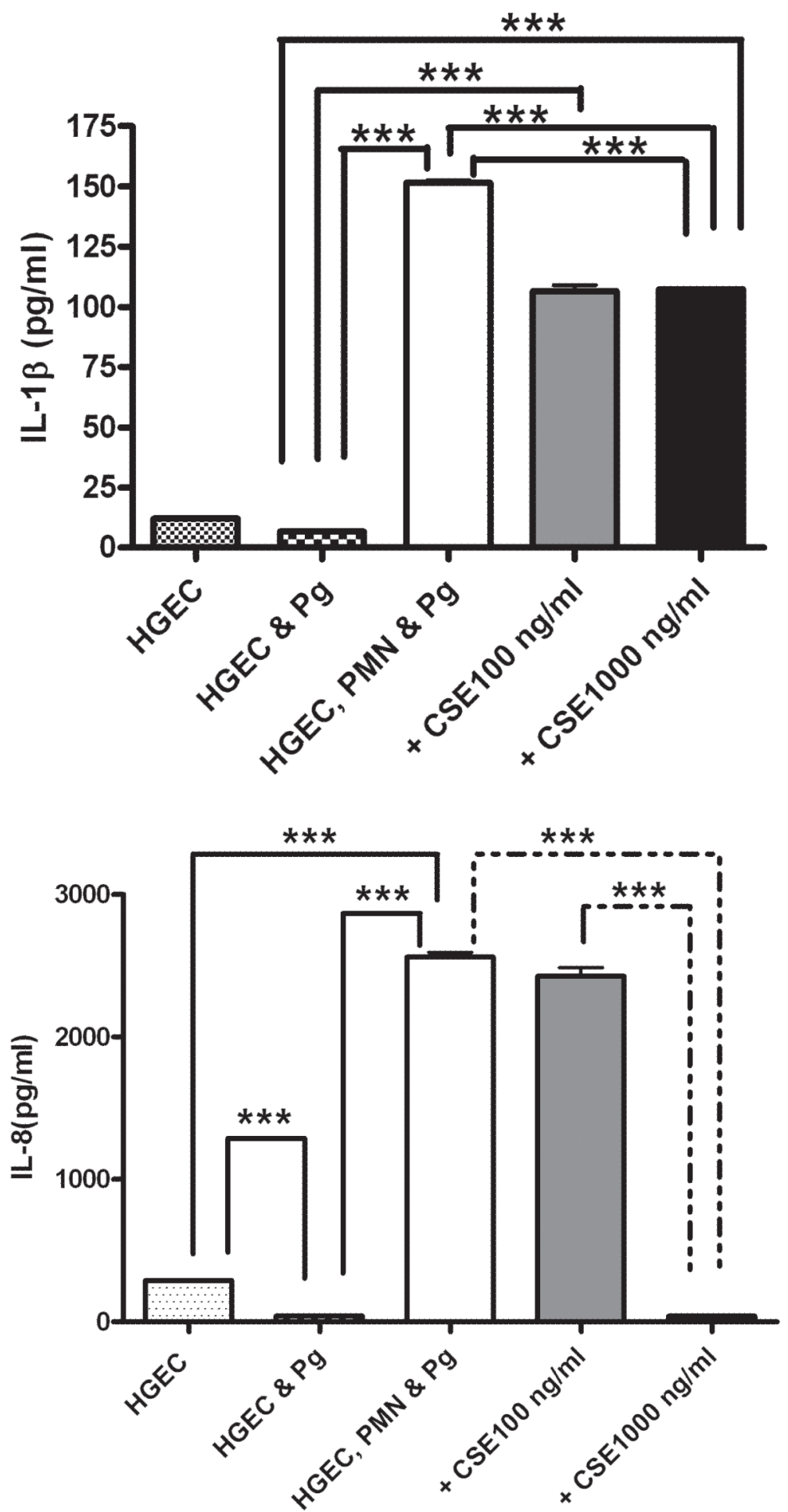

Figure 1. CSE suppresses the production of pro-inflammatory cytokines in a gingival crevice model

Human gingival epithelial cells were stimulated with $P$. gingivalis in the presence or absence of primary human neutrophils and CSE. HGEC: P. gingivalis: neutrophil ratio was 1:100:1. Concentrations of (A) IL-1 $\beta$ and (B) IL-8 in $24 \mathrm{hr}$ cell-free supernatants of $P$. gingivalis- 
stimulated HGECs in the presence or absence of neutrophils with or without CSE was measured by ELISA.

HGEC only (dotted bars); HGEC plus $P$. gingivalis (checked bars); HGEC plus $P$. gingivalis plus neutrophils (white bars); HGEC plus $P$. gingivalis plus neutrophils plus nicotine at 100 $\mathrm{ng} / \mathrm{ml}$ nicotine equivalency (grey bars); HGEC plus $P$. gingivalis plus neutrophils plus nicotine at $100 \mathrm{ng} / \mathrm{ml}$ nicotine equivalency (blackbars).

$* * * \mathrm{p}<0.001 ; * * \mathrm{p}<0.01$. 

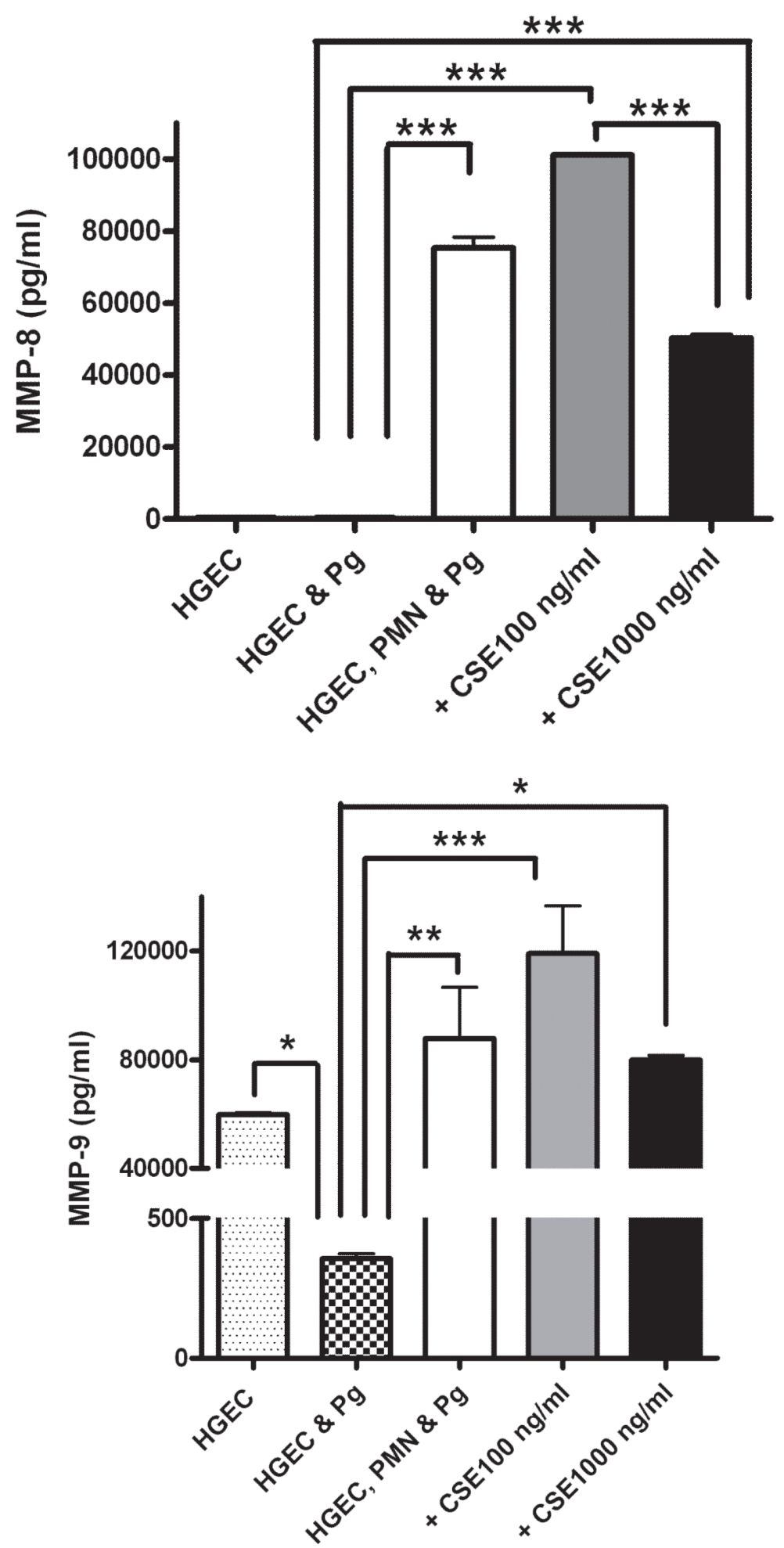

Figure 2. Burden of total MMP-8 and MMP-9 in a gingival crevice model Human gingival epithelial cells were stimulated with $P$. gingivalis in the presence or absence of primary human neutrophils and CSE. HGEC: $P$. gingivalis: neutrophil ratio was 1:100:1. Concentrations of (A) MMP-8 and (B) MMP-9 in $24 \mathrm{hr}$ cell-free supernatants of $P$. 
gingivalis-stimulated HGECs in the presence or absence of neutrophils with or without CSE was measured by ELISA.HGEC only (dotted bars); HGEC plus $P$. gingivalis (checked bars); HGEC plus $P$. gingivalis plus neutrophils (white bars); HGEC plus $P$. gingivalis plus neutrophils plus nicotine at $100 \mathrm{ng} / \mathrm{ml}$ nicotine equivalency (grey bars); HGEC plus $P$. gingivalis plus neutrophils plus nicotine at $100 \mathrm{ng} / \mathrm{ml}$ nicotine equivalency (blackbars). $* * * \mathrm{p}<0.001 ; * * \mathrm{p}<0.01 ; \mathrm{p}<0.05$ 

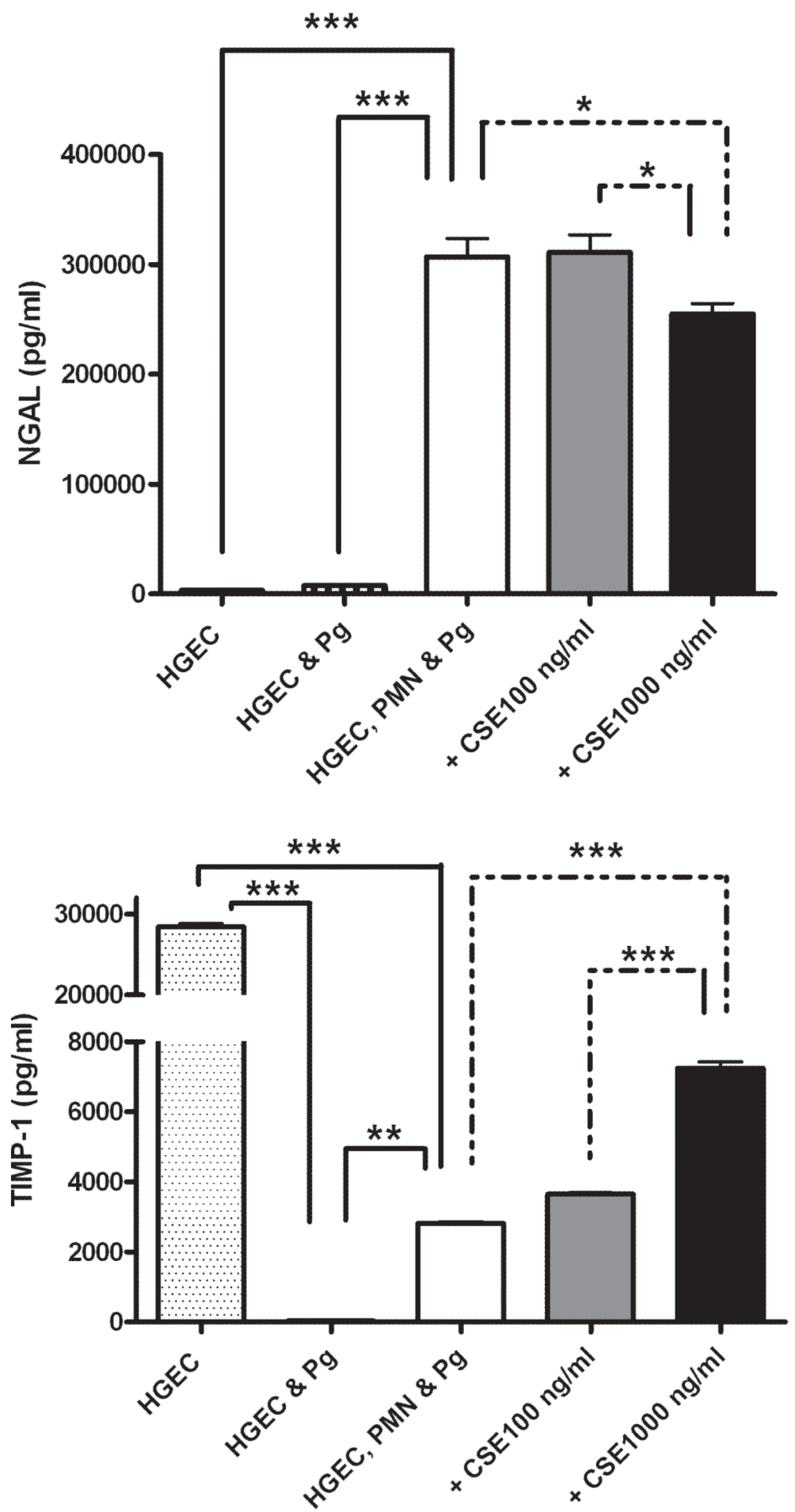

Figure 3. Production of MMP-modulators in a gingival crevice model Human gingival epithelial cells were stimulated with $P$. gingivalis in the presence or absence of primary human neutrophils and CSE. HGEC: $P$. gingivalis: neutrophil ratio was 1:100:1. Concentrations of (A) NGAL and (B) TIMP-1 in $24 \mathrm{hr}$ cell-free supernatants of $P$. gingivalis-stimulated HGECs in the presence or absence of neutrophils and CSEwas measured by ELISA. HGEC only (dotted bars); HGEC plus P. gingivalis (checked bars); 
HGEC plus $P$. gingivalis plus neutrophils (white bars); HGEC plus $P$. gingivalis plus neutrophils plus nicotine at $100 \mathrm{ng} / \mathrm{ml}$ nicotine equivalency (grey bars); HGEC plus $P$. gingivalis plus neutrophils plus nicotine at $100 \mathrm{ng} / \mathrm{ml}$ nicotine equivalency (blackbars). $* * * \mathrm{p}<0.001 ; * * \mathrm{p}<0.01 ; \mathrm{p}<0.05$. 


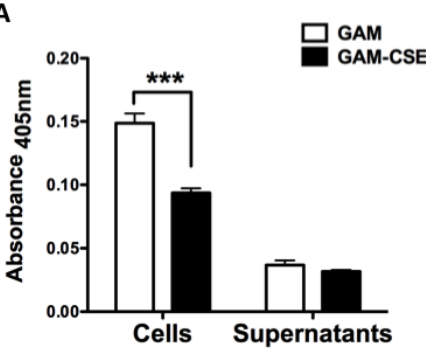

C

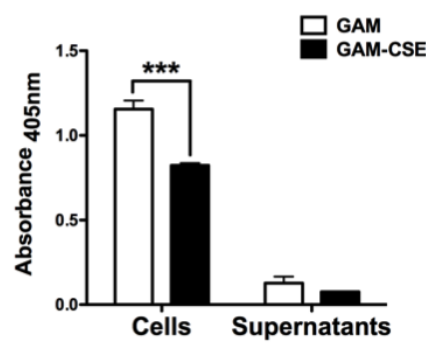

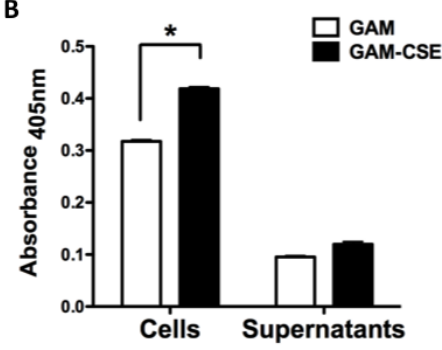

D

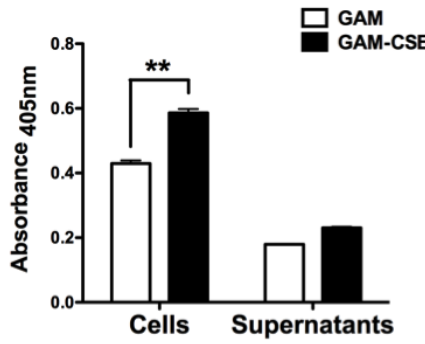

Figure 4. Influence of CSE on gingipain production is $P$. gingivalis strain-specific The influence of CSE on gingipain production by two strains of $P$. gingivalis was assessed. Total Kgp activity in control and CSE-exposed cultures of (A) P. gingivalis 33277 and (B) $P$. gingivalis $\mathrm{W} 83$ was determined by spectrophotometric analysis of acetyl-lysine- $p$ nitroanilide degradation. Total Rgp activity in control and CSE-exposed cultures of (C) $P$. gingivalis 33277 and (D) $P$. gingivalis W83 was determined by spectrophotometric analysis of $N$-a-benzoyl-D,L-arginine- $p$-nitroanilide degradation.

$\mathrm{p}<0.001 ; * * \mathrm{p}<0.01 ; \mathrm{p}<0.05$. 
A

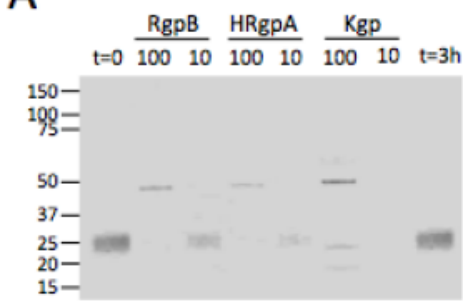

C

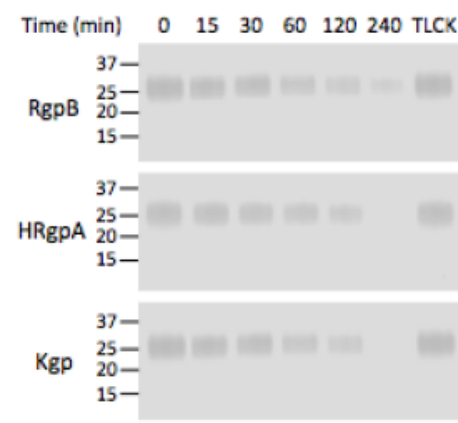

B

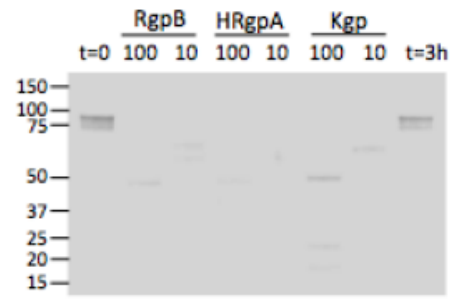

D

Time (min) $\quad 0 \quad 15 \quad 30 \quad 60 \quad 120 \quad 240$ TLCK

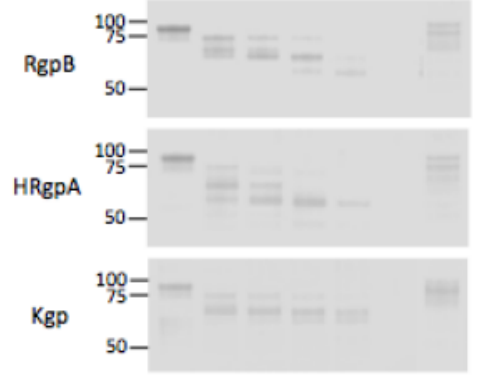

Figure 5. $P$. gingivalis gingipains degrade TIMP-1 and MMP-9

The ability of RgpA, HRgpA and Kgp gingipains to degrade TIMP-1 and MMP-9 was assessed. Recombinant TIMP-1 (A) or MMP-9 (B), $400 \mu \mathrm{g} / \mathrm{ml}$, were incubated with RgpA, HRgpA or Kgp (active proteases 10 and $100 \mathrm{nM}$ ) at $37^{\circ} \mathrm{C}$ in gingipain activity buffer with $10 \mathrm{mM}$ L-cysteine. After 3 hours, TLCK was added to block the reaction and residual proteins were visualized by SDS-PAGE under reducing conditions. The degradation of TIMP-1 (C) and MMP-9 (D) over 180 min was also monitored. TIMP-1 and MMP-9 incubated without gingipains served as negative controls. Typical digests are presented. 